\title{
Clinical Quiz: The Strawberry Coloured Breastmilk
}

\author{
Jamani NA ${ }^{\mathrm{a}}$, Puteri Shanaz JK ${ }^{\mathrm{b}}$ \\ ${ }^{a}$ Department of Family Medicine, Kulliyyah of Medicine, International Islamic University Malaysia, Jalan \\ Sultan Ahmad Shah, 25200 Kuantan Pahang \\ ${ }^{b}$ Department of Family Medicine, Faculty of Medicine and Health Sciences, Universiti Putra Malaysia, \\ 43400 Serdang, Selangor
}

\section{HISTORY}

A 33 year-old Para 1 complained of strawberry coloured breastmilk for 1 day duration while expressing her breastmilk on the left breast at work. She has been exclusively breastfeeding for the past 6 months and never encountered such incident before. She also felt pain over the left breast. However, there was no lump, nipple crack, abnormal nipple discharges or fever prior to that. She expressed her breastmilk using a double mechanical electric breastpump about five times a day lasting from 10 to 20 mintues per sesion since she returned to work.

On examination, her vital signs were normal and she was afebrile. Examination of the breasts revealed a lactating breast which were normal in size and shape. Her left breast showed a localized tenderness and was mildly swollen at the outer lower quadrant. No erythematous skin changes was noted. There was no palpable mass and no abnormal nipple discharges. A pink milk was seen when the nipple is expressed. The right breast and axillary region were normal.All investigations including full blood count, breast milk analysis, culture and sensitivity were normal.

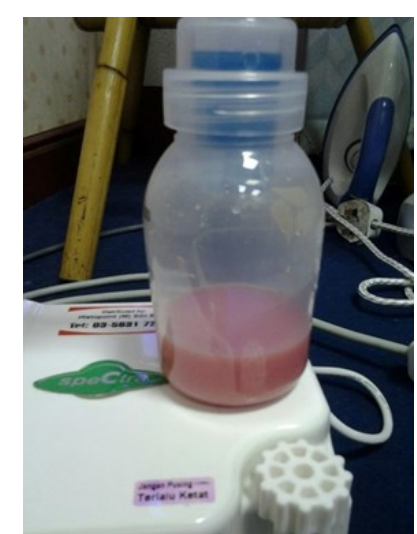

Figure 1. Blood stained breast milk

Corresponding author:

Nurjasmine Aida Jamani MD

(UKM), M.Med (Fam Med)

Department of Family Medicine,

Kulliyyah of Medicine,

International Islamic University Malaysia,

Jalan Sultan Ahmad Shah,

25200 Kuantan Pahang

Email: minaida@iium.edu.my

\section{Question 1}

What is the most likely diagnosis?
A. Breast abcess
B. Breast cancer
C. Intraductal papilloma
D. Mastitis
E. Rusty pipe syndrome

\section{Answer: D}

\section{Question 2}

What is the most appropriate management for this patient?

A. Advice to stop expressing her breastmilk

B. Apply topical antibiotic

C. Prescribe oral antibiotic

D. Refer for mammogram

E. Refer for ultrasound of the breast

\section{Answer: C}

\section{DISCUSSION}

Blood stained breastmilk is not uncommon but can be alarming to a nursing mother. Blood in breast milk can vary in appearance from pink, orange, red and brown. Blood stained breast milk can be due condition like rusty pipe syndrome, nipple and breast trauma, as well as intraductal papiloma. ${ }^{1}$ This patient is diagnosed to have mastitis of the left breast which intially started as plugged ducts evidenced by focal breast engorgement which, after repeated pumping, had caused trauma to the blood capilaries of the breast giving the appearance of pink milk. Lactation mastitis is an inflammatory condition of the breast which may or may not be accompanied by infection.

It is a clinical diagnosis and investigations are not indicated in the initial assessment. ${ }^{2}$ It can be caused by blocked milk ducts causing milk stasis which is a potential medium for bacteria growth. Mastitis is classically characterized by unilateral or bilateral breast pain or tenderness, swelling and segmental erythema. ${ }^{2}$ A painful, white, 1 -mm bleb may also be present on the nipple, which is thought to be an overgrowth of epithelium or an accumulation of particulate or fatty material. ${ }^{3}$ However, the signs and symptoms of infectious mastitis are difficult to distinguish from non-infectious mastitis. ${ }^{2}$ Mastitis 
can give rise to blood, mucus and pus in the milk. It must be treated promptly and adequately in order to prevent breast abcess and relapse. The main principle of treatment of mastitis is supportive counselling, effective milk removal, antibiotic therapy and adequate analgesics. ${ }^{2}$ Breastfeeding should be reinforced and is safe for the infant.

\section{REFERENCES}

1. Mohrbacher N. Breastfeeding Answers Made Simple: A guide for helping mothers. 1st Edition . Hale Publishing, 702-703. Hale Publisher; 2010. 702-703 p.

2. World Health Organization. Mastitis [Internet]. 2000 [cited 2016 Jul 26]. Available from:

http://whqlibdoc.who.int/hq/2000/ WHO_FCH_CAH_00.13.pdf.

3. Spencer J. P. Management of Mastitis in Breastfeeding Women. Am Fam Physician. 2008 Sep 15;78(6):727-731. 
